\title{
Enhanced WOA and Modular Neural Network for Severity Analysis of Tuberculosis
}

\author{
Chithra R S \\ Dept. of Computer Science Engineering \\ Ponjesly College of Engineering \\ Nagercoil, Tamil Nadu, India \\ chithirarsrs@gmail.com
}

\author{
Jagatheeswari $\mathbf{P}$ \\ Dept. of Electrical and Electronics Engineering \\ Ponjesly College of Engineering \\ Nagercoil, Tamil Nadu, India
}

\begin{abstract}
Generally, Tuberculosis (TB) is an extremely infectious disease and it is a significant medical issue everywhere throughout the globe. The exact recognition of TB is the main concern faced by the majority of conventional algorithms. Hence, this paper addresses these problems and presented a successful method for recognizing TB utilizing the modular neural network. Moreover, for transforming the RGB image to LUV space, the color space transformation is utilized. At that point, adaptive thresholding is done for image segmentation and several features, such as density, coverage, color histogram, length, area, and texture features, are extracted to enable effectual classification. Subsequent to the feature extraction, the size of the features is decreased by exploiting Principal Component Analysis (PCA). For the classification, the extracted features are exposed to Whale Optimization Algorithm-based Convolutional Neural Network (WOA-CNN). Subsequently, the image level features, such as bacilli area, bacilli count, scattering coefficients and skeleton features are considered to do severity detection utilizing proposed Enhanced Whale Optimization Algorithm-based Modular Neural Network (EWOA-MNN). In conclusion, the inflection level is resolved to utilize density, entropy, and detection percentage. The proposed method is modeled by enhancing the WOA method.
\end{abstract}

Keywords: Tuberculosis; Thresholding; PCA; Neural Network; Optimization Algorithm

\section{Introduction}

In spite of the developments in medicine and the growth of non-expensive and effectual treatments, tuberculosis (TB) is considered as the most killers global. In recent years, it is evaluated 8.6 million new TB cases happened; also 1.3 million people expire from this disease [1]. TB is extremely contagious, and the gasp of some bacteria (Mycobacteria tuberculosis) is sufficient for a human being to turn into infected. Therefore, early diagnosis and detection are important to prevent its dissemination. Even though numerous tests for TB diagnosis, whichever latent (for instance: interferon-gamma release assay or Mantoux test) otherwise active (for instance: Xpert MTB/RIF or sputum culture), subsist. Moreover, their application is typically hampered using the medium to long-time necessary to procedure a sample, their difficulty or their relative maximum cost. However, the regions with the uppermost TB burden in the world are considered which are between those with the lowly economic resources [6]. In this circumstance, chest radiography is the topic of transformed attention as a corresponding tool in TB diagnostic approaches, as it can be much faster and cheaper, and can allow identification of TB suspects with the uppermost risk of disease. Regrettably, evaluating Chest Radiographs (CXRs) needs specialized personnel which is not always obtainable that is the main impediment in the direction of their employ, particularly in the aforesaid resource-constrained areas [11] [12].

In worldwide, TB is the main widespread reason for death from a transmittable disease, in accordance with HIV, with a mortality rate of above 1.2 million people [8]. Generally, TB is a contagious disease. Typically through the bacillus Mycobacterium TB caused the lung. It spreads by the air while people with active TB give out sneezing, coughing, or else infectious bacteria. In Southeast and Africa, TB is extensively malnutrition and poverty to minimize resistance to the disease most frequently. Additionally, opportunistic infections in immune cooperate to increase the issue of AIDS/HIV patients [9]. The growing occurrence of multi-drug opposed to TB was new, and a vital requirement for economical screening equipment to have to check the growth of treatment. For the treatment of TB, numerous antibiotics are obtainable. Whereas the mortality rate increases, and if left untreated, the antibiotic treatment enhances the probability of endurance. 
The conservative diagnosis of TB includes clinical investigation, bacteriological examination, and chest x-ray. In sputum samples, the bacteriological test engages direct observation of acid-fast bacilli and additional processing of sputum samples for culture [7]. Despite the minimum specificity and sensitivity of sputum microscopy, in addition to the restriction in its capability to distinguish Mycobacterium TB complex (MTBC) from other Nontuberculosis Mycobacteria (NTM), in developing countries sputum microscopy is still extensively exploited. Bacteriological culture, whereas considered a gold standard examination for TB diagnosis, needs infrastructure, skilled manpower, and 6-8 weeks to obtain results. In the alike vein, molecular-based speed diagnostic tools, like the Hain line probe assay (Hain life sciences $\mathrm{GmbH}$ ) and Genexpert (Cepheid, CA), may not be suitable for regular diagnosis of TB in developing countries because of its high cost and inadequate laboratory infrastructure [17] [18].

As of now, the primary technique for research facility finding for tuberculosis is the utilization of a microbiological test, for example, sputum solid culture, a sputum smear, else liquid culture system (for instance MGIT 960). The exactness of the test results can be impacted by different elements, such as reagent quality, specimen status, and operation proficiency [13]. The consequences of these tests are utilized to decide the right medicine and to assess infection transmission and prediction. In this manner, quality control of tuberculosis the indicative tests is significant. To enhance the test quality crosswise over research centers, standard approaches have been discharged; these rules present a progression of quality control protocols for the design, culture medium, hardware, and reagents utilized in M. tuberculosis research facilities [14] [32]. Be that as it may, there is no generally accepted protocol for the quality control of tuberculosis research center test results [15] [16].

The main contribution of this paper is to presents an algorithm, called Enhanced Whale Optimization algorithm-based Modular Neural Network (EWOA-MNN), for severity infection and detection level identification utilizing sputum smear images. Subsequent to severity detection, the infection level is recognized utilizing the density, entropy, and detection percentage.

\section{Literature Review}

In 2019, Jie $\mathrm{Wu}$ et al, [1], worked on tuberculosis detection laboratory cross-contamination utilizing entire-genome sequencing. During the drug resistance observation, a sum of $22 \mathrm{M}$. tuberculosis strains with maximum genotypic homology from one hospital was gathered. Epidemiological examination and genome sequencing were led to decide the event of cross-contamination. From the single institute, an increasing extent of M. tuberculosis strains with genotypic homology which shared a similar procedure time period was in all likelihood brought about the cross contagion. The entire genome sequencing examination can assist to decide the event of cross contagion.

In 2019, Ellen Jo Baron et al [2], worked on TB, which keeps on being a noteworthy reason for mortality and morbidity around the world. In clinical specimens, quick detection of Mycobacterium TB straightforwardly utilizing nucleic acid intensification examinations empowers tainted patients to be set on fitting treatment much earlier than while the consequences of regular culture techniques were utilized. Also, the accessibility of fast results encourages disease control measures to interfere with the transmission of TB in medicinal services settings. The time of economically accessible atomic diagnostics was started 25 years prior to the detection of M. tuberculosis and currently encompasses numerous examines that can distinguish life forms in the M. tuberculosis complex. Additionally, a few tests distinguish chromosomal changes related to antimicrobial protection from additional refine remedial systems near the beginning in the course of the disease. NAATs had reformed the finding of TB over the world and have turned into the normal of consideration in numerous high-trouble creating nations

In 2019, Jeewan Thapa et al [3], studied the development of a basic Visual Methyl Green (MeG) on the basis of dry Loop-Mediated Isothermal Amplification (LAMP) strategy for early recognition of Mycobacterium tuberculosis (MTB) from clinical examples. Moreover, they had distinguished MeG as a marker of a positive LAMP response, where a positive response gave Bluegreen shading while a negative response was dismal. By exploiting Nepalese clinical samples, the MeG MTB-LAMP framework was additionally simplified by drying all reagents for convenience and was subsequently examined for its capacity to analyze TB straightforwardly.

In 2019, Karoline Wagner et al [4], worked on a line probe that examines the empowerment of the fast discovery of drug-resistant Mycobacterium TB openly from clinical samples. Moreover, this investigation demonstrates which characterizing threshold values in microscopic assessment or PCR discovery of M. TB enhances line probe assay efficiency, along these lines evading expenses and loss of time because of superfluous sub-atomic testing.

In 2019, Reza Kamali Kakhki et al [5], worked on the recognition of TB, which was the vital strides for TB control. In spite of the fact that the sensitivity of traditional algorithms such as Lowenstein Jensen (LJ) culture and direct recoloring was very low, molecular techniques such as Polymerase Chain 
Reaction (PCR) were increasingly sensitive and be considered as valuable devices for quick discovery of tuberculosis. Different genes such as mpb64 and IS6110 were utilized as the objective of detection for M. tuberculosis, yet additional investigate were expected to locate the majority explicit objectives. The Short-Chain dehydrogenases/Reductases family (SDR) was an enormous group of NAD- or NADPdependent oxidoreductase enzymes that were available in all M. TB strains. The huge piece of SDR groupings in TB was totally preserved and not the same as non-TB mycobacterium.

\section{Proposed EWOA-MNN for Severity Analysis of TB}

In this section, the proposed algorithm for the diagnosis of TB from microscopic sputum smear images with infection level recognition is presented. Numerous conventional algorithms present high time for calculation, which present results with minimum accuracy and also costly. At first, for segmenting the image, the adaptive thresholding algorithm is exploited subsequent to the color space transformation. For feature extraction, the segments attained from the adaptive thresholding are exploited. Subsequently, the extracted features are exposed to WOA-CNN for classifying the image into 3 types, such as bacilli, no bacilli, and overlapping bacilli. The proposed EWOA-Modular neural network algorithm is exploited, for the severity analysis, Here, the modular network is trained by utilizing enhanced WOA method. Subsequently, area, the number of bacilli, skeleton features and scattering coefficient of the segmented image are extracted and subjected to the proposed EWOA-Modular Neural Network. The algorithm is effectual, which processes a large number of data, and thus, the death rate is then minimized using the adapting automatic classification of sputum images. Fig 1 exhibits the schematic illustration of the proposed EWOA-MNN for severity detection.

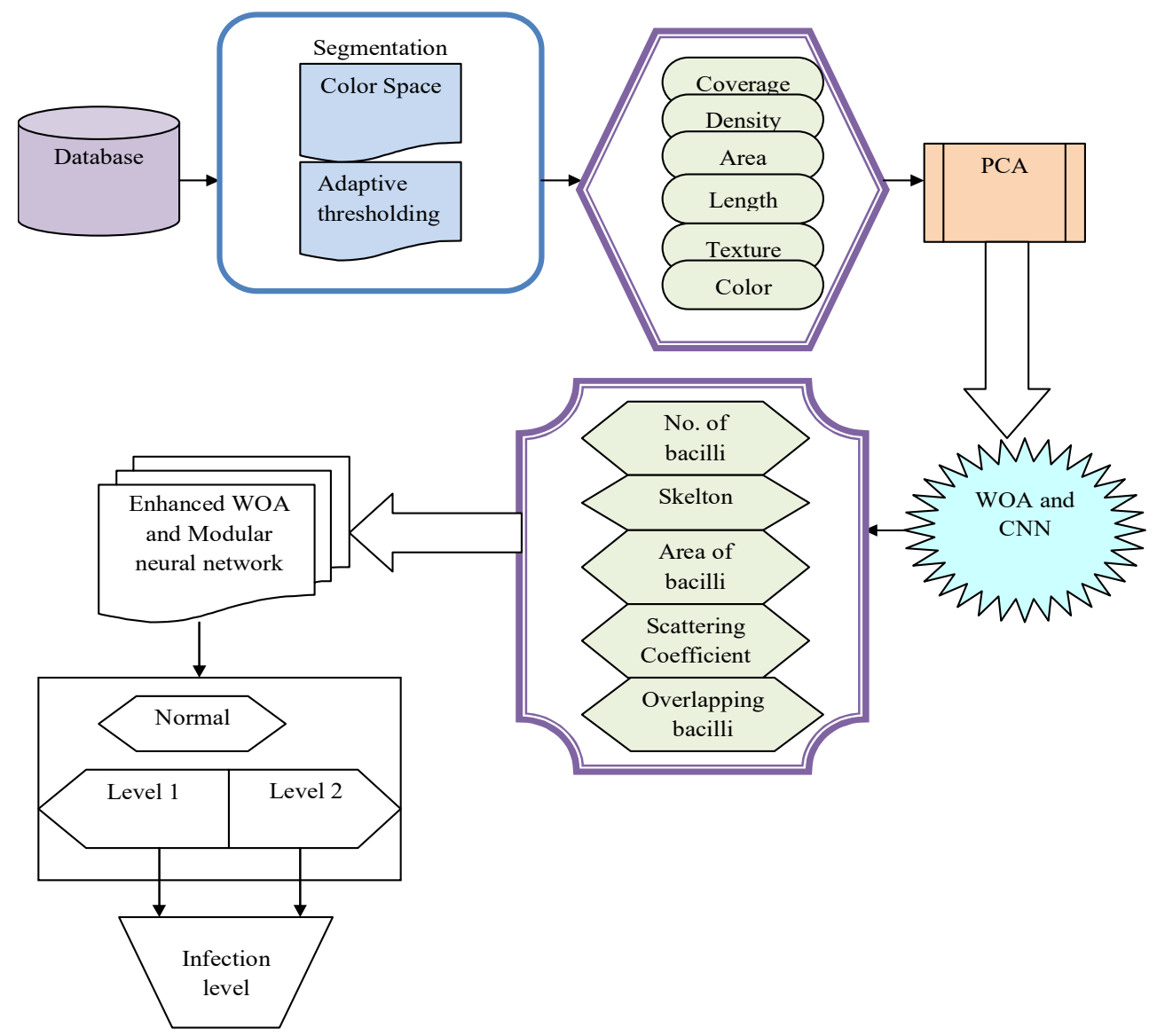

Fig. 1. Schematic diagram dor severity detection using proposed EWOA-MNN

Let us consider a database $\mathrm{DB}$ with dimension $\mathrm{L}_{\mathrm{c}} \times \mathrm{M}_{\mathrm{d}}$ comprising 's' sputum, which is stated in eq. (1).

$$
\mathrm{DB}=\left\{\mathrm{DB}_{1}, \mathrm{DB}_{2}, \ldots, \mathrm{DB}_{\mathrm{i}}, \ldots, \mathrm{DB}_{\mathrm{s}}\right\} ; 1 \leq \mathrm{i} \leq \mathrm{s}
$$

In eq. (1), $\mathrm{DB}_{\mathrm{i}}$ indicates the $\mathrm{i}^{\text {th }}$ input image where. 


\subsection{Adaptive Threshold Method for Segmentation of Images}

\section{a) Transformation of color space:}

The color space transformation is modified with the absence of difficulty, and the description in a standard way. Moreover, the motivation for doing color space transformation is to produce inputs with general color spaces prior to procedure implementation. In general, from RGB information, the color spaces are derived. An RGB image comprises three layers which include green, red, and blue colors and it is a three-dimensional matrix. Moreover, the intensity is considered as a significant parameter to be contemplated to distinguish the edges and to procedure the image. The RGB image is indicated as $\mathrm{DB}_{\mathrm{i}}^{\mathrm{A}}$ and is altered to LUV space indicated as $\mathrm{D}_{\mathrm{i}}^{\mathrm{P}}$.

\section{b) Adaptive Thresholding:}

By exploiting the binary image format the image thresholding is considered as a procedure, in order to classify the pixels as light or dark. In contrast, the adaptive thresholding contemplates the spatial variations in illumination. In [19], the adaptive thresholding is exploited in order to address the deviation by exploiting varying threshold values for each pixel from the image. For producing the segments the Q spaces are considered from the original image. Moreover, the adaptive thresholding is done to present segments of a digital image with distinct image features in that one feature is intensity levels. When processing variations in the illumination the adaptive thresholding presents superior quality. At first, the fundamental image is recognized, and subsequently, the average $(\mathrm{N} \times \mathrm{N})$ window of an individual pixel is computed. If the value of the pixel is lesser than the average of $(\mathrm{N} \times \mathrm{N})$ neighbors, subsequently the pixel is black else the pixel is black. The segments are produced so that adaptive thresholding is stated in eq. (2).

$$
\mathrm{L}^{\mathrm{i}}=\left\{\mathrm{L}_{1}^{\mathrm{i}}, \mathrm{L}_{2}^{\mathrm{i}}, \ldots, \mathrm{L}_{\mathrm{m}}^{\mathrm{i}}, \ldots \mathrm{L}_{\mathrm{r}}^{\mathrm{i}}\right\}
$$

In eq. (2), $\mathrm{L}_{\mathrm{m}}^{\mathrm{i}}$ indicates the $\mathrm{m}^{\text {th }}$ segment of $\mathrm{i}^{\text {th }}$ image, and from the $\mathrm{i}^{\text {th }}$ image ' $\mathrm{r}$ ' indicates the total segments produced.

\subsection{Feature Extraction from the Segmented Image}

From the input image $\mathrm{DB}_{\mathrm{i}}$, the segments obtained from an adaptive thresholding algorithm are recognized and it is exploited for feature extraction. Moreover, the feature extraction is done for effectual classification of sputum images comprised in the database and for recognizing the persons with TB and hence, the severity level is decided.

\section{a) Coverage:}

By exploiting the image coverage and shape, the coverage factor is exploited to classify the person. The average of the extracted important points is calculated for attaining the coverage feature. In order to denote the location of the object, the average value is exploited and the coverage factor is used to measure the length, and the width of the bacilli. $\mathrm{T}_{1}^{\mathrm{m}, \mathrm{i}}$ indicate the coverage feature of $\mathrm{q}^{\text {th }}$ segment in $\mathrm{i}^{\text {th }}$ image with dimension $[1 \times 1]$.

\section{b) Density:}

The procedure exploited to compute the density feature of the segment is called as zoning [20]. Initially, the segments are classified into zones with fixed sizes and for each zone, the features are extracted. $\mathrm{T}_{2}^{\mathrm{m}, \mathrm{i}}$ indicates the density feature of the $\mathrm{m}^{\text {th }}$ segment in the $\mathrm{k}^{\text {th }}$ image and the dimension is stated as $[1 \times 1]$.Eq. (3) indicates the density feature.

$$
\mathrm{T}_{2}^{\mathrm{m}, \mathrm{i}}=\frac{\left|\mathrm{L}_{\mathrm{m}}^{\mathrm{f}}\right|}{\left|\mathrm{L}_{\mathrm{m}}\right|}
$$

In eq. (3), $\left|\mathrm{L}_{\mathrm{m}}^{\mathrm{f}}\right|$ indicates the total foreground pixels contained in the $\mathrm{m}^{\text {th }}$ segment and $\left|\mathrm{L}_{\mathrm{m}}\right|$ indicates the total numbers of the pixels in the $\mathrm{m}^{\text {th }}$ segment.

\section{c) Color Histogram}

In [21], the partitioning of the color composition comprised in the segments is indicated using the color histogram. If the color space is higher, the pixel is partitioned into bins in order to indicate the color histogram of segments. The histogram exhibits the probability distribution of intensity levels regarding the image. Eq. (4) is used to represents the histogram probability.

$$
l(d)=\frac{L_{d}}{x}
$$


In eq. (4), ' $\mathrm{x}$ ' indicates the total number of pixels in the segment, and $\mathrm{L}_{\mathrm{d}}$ indicates the total pixels at the gray level. The color histogram feature is shown as $\mathrm{T}_{3}^{\mathrm{m}, \mathrm{i}}$ and its dimension is indicated as $[3 \times \delta]$. Eq. (5) is used to formulate the color histogram feature.

$$
\mathrm{T}_{3}^{\mathrm{m}, \mathrm{i}}=\left\{\left.1^{\mathrm{R}}(\mathrm{d})\left\|1^{\mathrm{g}}(\mathrm{d})\right\|\right|^{\mathrm{D}}(\mathrm{d})\right\}
$$

In eq. (5), $\mathrm{l}^{\mathrm{R}}(\mathrm{d}), \mathrm{l}^{\mathrm{g}}(\mathrm{d})$, and $\mathrm{l}^{\mathrm{D}}(\mathrm{d})$ indicate the histogram probabilities of $\mathrm{R}, \mathrm{G}$, and $\mathrm{B}$ bands, correspondingly, and $\mathrm{d}$ indicates the total pixels at the grey level and the pixel values range from 0 to 255 .

\section{d) Texture Features}

In [22], the Local Directional Pattern (LDP) is contemplated as improved Local Pattern Descriptor (LPD), the combines a directional component by adapting Kirsch compass kernels. The LDP is less at risk to noise as evaluated to conventional LBP operators.

$$
\operatorname{LDP}_{\mathrm{b}}\left(\mathrm{c}_{\mathrm{n}}, \mathrm{d}_{\mathrm{n}}\right)=\sum_{\mathrm{x}=0}^{7} \mathrm{u}\left(\mathrm{h}_{\mathrm{x}}-\mathrm{h}_{\mathrm{b}}\right) \cdot 2^{\mathrm{x}}
$$

In eq. (6), $\left(\mathrm{c}_{\mathrm{n}}, \mathrm{d}_{\mathrm{n}}\right)$ indicates pixel position, bindicates the directional bit responses, $\mathrm{x}$ indicates neighborhood pixel number, $h_{b}$ indicates $b^{\text {th }}$ directional response and $h_{x}$ indicates Kirsch masks.

$$
\text { where, } u(c)=\left\{\begin{array}{l}
1 \text { if } c \geq 0 \\
0 \text { otherwise }
\end{array}\right\}
$$

Here, Local Gradient Pattern (LGP) [23], which modifies the gradient value of the 8 pixels, is also contemplated. The gradient values and center pixel are computed based on absolute difference regarding the neighboring pixel intensity and center pixel intensity. With the average of gradient values of neighboring pixels, the threshold is computed and the kernel is modified to calculate the neighbor pixels for center pixel. The neighboring pixel value is one if the gradient pixel is higher than the threshold or the value leads to be 0. Eq. (8) and (9) formulates the LGP operator.

$$
\begin{aligned}
& \mathrm{T}_{4}^{\mathrm{m}, \mathrm{i}}=\mathrm{LGP}_{\delta, \alpha}^{\mathrm{vi}, \mathrm{u}^{\mathrm{i}}}=\sum_{\mathrm{n}=0}^{\alpha-1} \eta\left[\bar{\sigma}_{\mathrm{n}}-\bar{\sigma}^{*}\right] \times 2^{\mathrm{n}} \\
& \xi(\mathrm{s})=\left\{\begin{array}{lll}
0 & ; & \mathrm{s}<0 \\
1 & ; & \text { otherwise }
\end{array}\right.
\end{aligned}
$$

In eq. (8), $\bar{\sigma}_{\mathrm{n}}$ denotes the gradient value between the center pixels and $\bar{\sigma}^{*}$ denotes the average of gradient values $\left(\lambda^{*}\right)$ positioned at $\left(\mathrm{v}^{\mathrm{i}}, \mathrm{u}^{\mathrm{i}}\right)$ and it's neighboring pixels $(\lambda)$ and it is stated in eq. (10)

$$
\bar{\mu}_{n}=\left|\lambda-\lambda^{*}\right|
$$

By starting the thresholding, the LGB is modified to produce codes by exploiting the absolute intensity difference with a locally adapted threshold $\lambda^{*}$. The LGP generates the invariant patterns by exploiting the gradient difference and LGP is not affected because of the local color variations. The dimension of color histograms is indicated as $[3 \times \gamma]$ where ' $\gamma$ ' signifies the bins.

\section{e) Bacilli area}

In the segments, the bacilli area is stated as the area covered exploiting the bacilli and for each segment, the area covered with bacilli is extracted and this area is indicated as $\mathrm{T}_{5}^{\mathrm{m}, \mathrm{i}}$ and its dimension is stated as $[1 \times 1]$.

\section{f) Bacilli length}

The bacilli length is measured to decide whether a bacillus is the overlapping bacilli or not from each segmented section of the image. Moreover, the length of bacilli in the $\mathrm{m}^{\text {th }}$ segment of the $i^{\text {th }}$ image is indicated as $\mathrm{T}_{6}^{\mathrm{m}, \mathrm{i}}$.

\subsection{Feature Vector Representation}

For the $\mathrm{m}^{\text {th }}$ segment, the segment features include the bacilli area, color histogram, texture, density, length, and coverage of bacilli are gathered to show the segment features. To classify the patients the features are exposed to the classifier based upon its overlapping bacilli or bacilli availability. Eq. (11) states the feature vector of the $\mathrm{m}^{\text {th }}$ segment.

$$
\mathrm{T}\left[\mathrm{L}_{\mathrm{m}}^{\mathrm{i}}\right]=\left\{\mathrm{T}_{1}^{\mathrm{m}, \mathrm{i}}, \mathrm{T}_{2}^{\mathrm{m}, \mathrm{i}}, \mathrm{T}_{3}^{\mathrm{m}, \mathrm{i}}, \mathrm{T}_{4}^{\mathrm{m}, \mathrm{i}}, \mathrm{T}_{5}^{\mathrm{m}, \mathrm{i}}, \mathrm{T}_{6}^{\mathrm{m}, \mathrm{i}}\right\}
$$


The dimension of the feature vector is indicated as $[1 \times(4+6 \kappa)]$ and the dimension features for the ' $r$ ' segments are indicated as $[\mathrm{r} \times(4+6 \kappa)]$.

\subsection{PCA for Dimension Reduction}

In [24], PCA is an approach that uses a linear transformation to model a clarified dataset to handle the features of the original data. Assume that the original matrix comprises of qdimensions and $j$ observations and it needs to minimize the dimensionality into $d$ dimensional subspace and the transformation is stated in eq. (12).

$$
\mathrm{T}=\mathrm{M}^{\prime} \mathrm{I}
$$

In eq. (12), $\mathrm{M}_{\mathrm{q} \times \mathrm{b}}$ indicates the projection matrix that is obtained from transpose, and comprises c eigenvectors with eigenvalues, and $\mathrm{I}_{\mathrm{q} \times \mathrm{j}}$ denotes the mean-centered data matrix.

\subsection{WOA-CNN Model for Patient Classification}

In this section, the WOA-CNN is used for the classification to recognize the TB patients. The features extracted are exploited as the input to the EWOA-MNN, in which WOA method is used to train the CNN to decide the optimal. WOA approach is modeled [29] that is a meta-heuristic population-based optimization approach that is easy to develop. Fig 2 shows the schematic diagram of the CNN model.

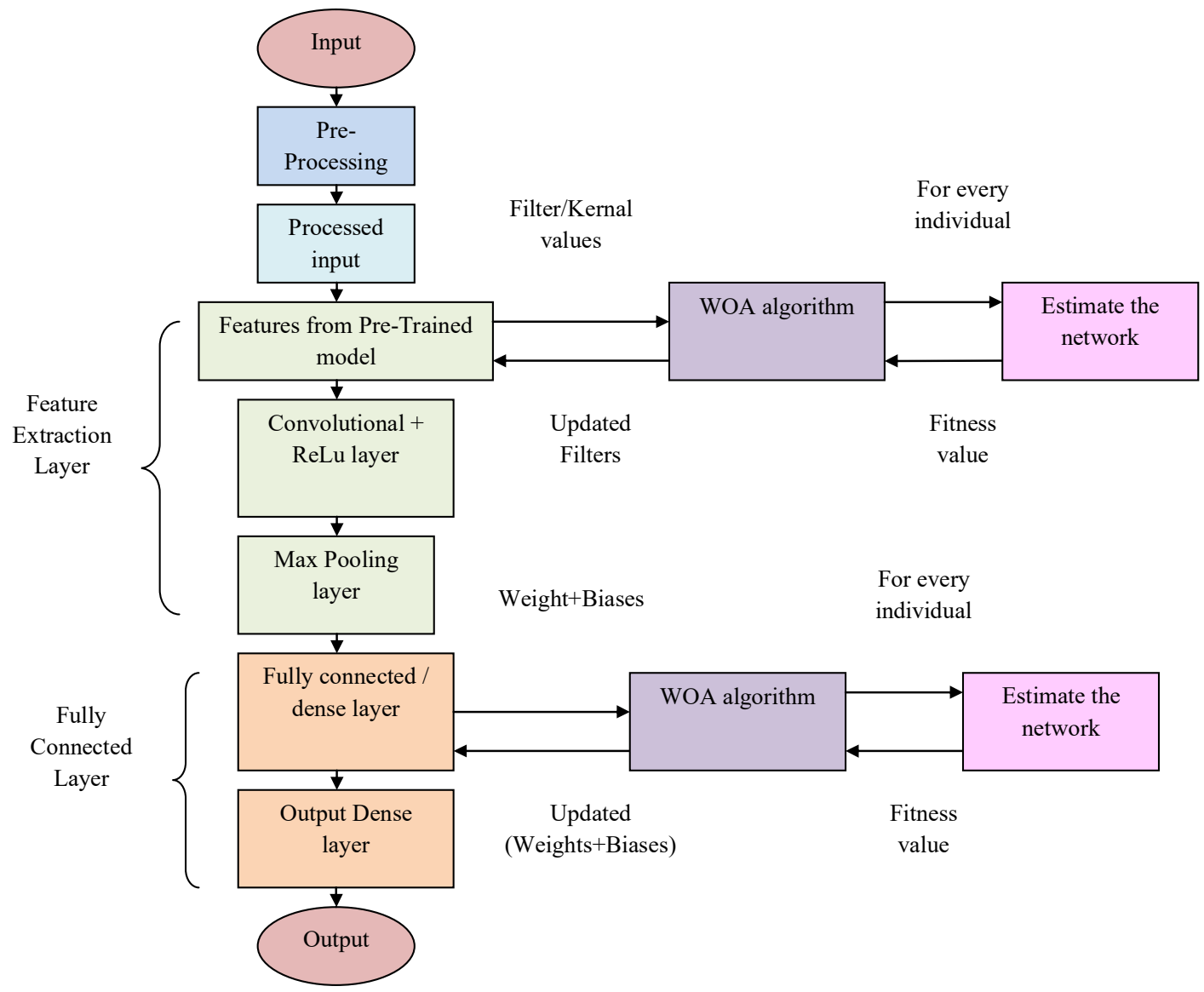

Fig. 2. Diagrammatic representation of the CNN

\subsection{Feature Extraction for Severity Analysis}

In this section, for analyzing some features that are extracted, the severity of the disease is stated. Moreover, the features, such as overlapping bacilli, number of bacilli, area of bacilli, in conjunction with skeleton feature and scattering coefficients are extracted from the segmented images. The number of bacilli is calculated by taking into consideration of the count of the number of pixels occurred using the bacilli, when the overlapped bacilli are stated as the ratio of the total area regarding the average area of one bacilli. Below, the other features are described: 
Skeleton feature: In [25], the skeleton feature uses skeleton pruning that is exploited to evade bacilli of trivial shape parts from the bacilli image. Hence, the skeleton point is generated by exploiting a maxdisc that comprises 2 touching points to shape the contour. In eq. (14), the pruning process is described.

$$
\kappa\left(\theta_{1}, \theta_{2}\right)=\frac{\chi\left(\theta_{1}, \theta_{2}\right) \ell\left(\theta_{1}\right) \ell\left(\theta_{2}\right)}{\ell\left(\theta_{1}\right)+\ell\left(\theta_{2}\right)}
$$

In eq. (14), $\chi\left(\theta_{1}, \theta_{2}\right)$ indicates the angle of the corner comprising $\theta_{1}$ and $\theta_{2}, \ell\left(\theta_{2}\right)$ and $\ell\left(\theta_{1}\right)$ indicates the length function normalized by exploiting total length.

Scattering Transform: In [26], the scattering coefficient states a texture feature that uses a Morlet wavelet for multiple orientation and scales. The scattering coefficient with maximum order is used to offer high sturdy features. From the image, these features dismiss the maximum frequency components. Moreover, the high-frequency bandpass filters are used in order to deal with high-frequency components. The scattering wavelet coefficient of order a is used at each layer of the network is represented in eq. (14).

$$
\gamma_{\mathrm{a}} \mathrm{D}(\mathrm{o}, \varphi)=||\left|\mathrm{D} * \phi \pi_{1}\right|{ }^{*} \phi \pi_{2}|, \ldots,|{ }^{*} \phi \pi_{v}\left|, \ldots,{ }^{*} \phi \pi_{\mathrm{a}}\right|{ }^{*} \varphi_{\delta}
$$

In eq. (14), D indicates the image, $\phi \tau_{\mathrm{a}}$ denotes ordered wavelet set and $\phi \pi_{1}\left|{ }^{*} \phi \pi_{2}\right|, \ldots,\left|{ }^{*} \phi \pi_{v}\right|, \ldots,{ }^{*} \phi \pi_{\mathrm{a}} \mid$ indicates wavelet modulus coefficients.

The feature vector is modeled by exploiting the features produced using the overlapping bacilli, a number of bacilli, area of bacilli, skeleton features and scattering coefficients is indicated using eq. (15).

$$
\mathrm{V}=\left[\mathrm{N}_{\mathrm{B}}, \mathrm{A}_{\mathrm{B}}, \mathrm{O}_{\mathrm{B}}, \kappa, \chi\right]
$$

In eq. (15), $A_{B}$ indicates the area of bacilli, $N_{B}$ indicates the number of bacilli $\mathrm{O}_{B}$ represents the overlapping bacilli, $\chi$ indicates the scattering coefficient and $\kappa$ indicates the skeleton feature. For the severity analysis, the feature vector is modeled in order to form the input to the EWOA-MNN.

\section{Proposed EWOA-MNN Methodology for Severity Analysis}

\subsection{Modular Neural Networks}

In [27], MNN is considered as the model which is exploited for classification, and identification. A modular NN can be inspected as a set of monolithic NNs [28] which gives out with a part of an issue, and subsequently their individual results are amalgamated by an integration unit to form a global solution to the entire issue. The major objective is that a complex issue can be partitioned into easier sub-issues which can be resolved by easier $\mathrm{NN}$ and subsequently the total solution will be an integration of the outputs of the easy monolithic NN. Fig 3 exhibits the block diagram of a Modular Neural Network.

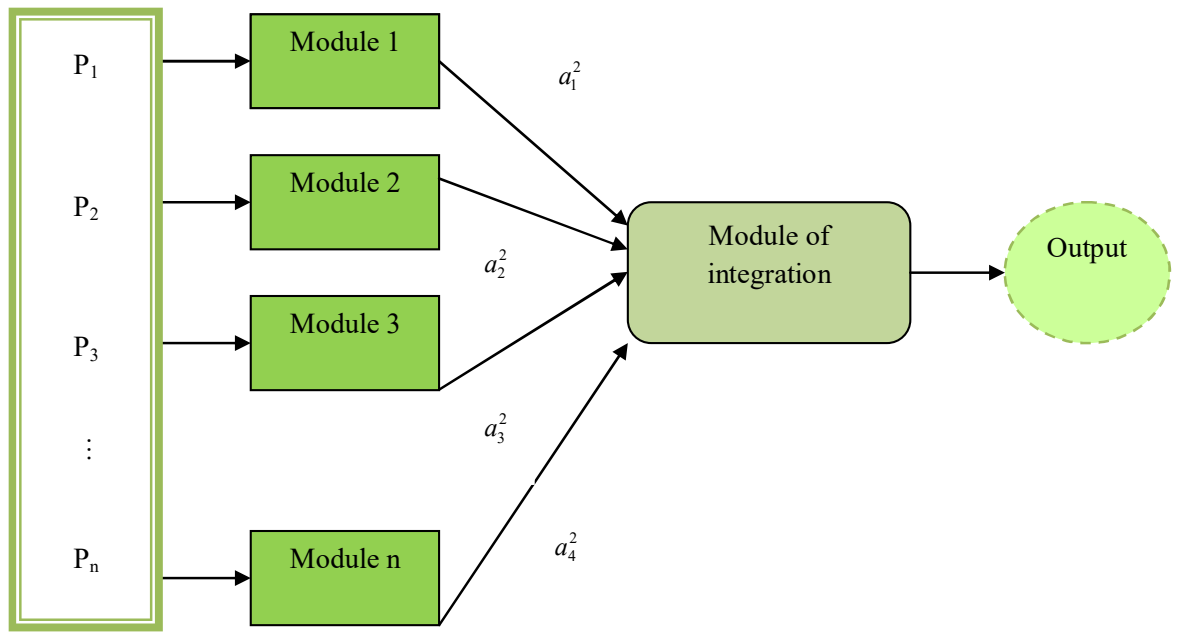

Fig. 3. Block diagram of Modular Neural Network

For the ANN, the Multilayer Perceptron and the result of the second layer are $\operatorname{an}_{\mathrm{i}}^{2}$, (for $\mathrm{i} \in\{1, \ldots, \mathrm{n}\}$ ) whereas $\mathrm{n}$ indicates the maximal number of modules.

Based on the following equation the output $\mathrm{OP}$ is computed that essentially do a weighted combination of the module outputs: 


$$
\mathrm{OP}=\frac{\sum_{\mathrm{i}=1}^{\mathrm{n}} \mathrm{an}_{\mathrm{i}}^{2} \mathrm{~h}_{\mathrm{i}} \mathrm{k}_{\mathrm{i}}}{\sum_{\mathrm{i}=1}^{\mathrm{n}} \mathrm{h}_{\mathrm{i}}}
$$

In eq. (16) $\mathrm{an}_{\mathrm{i}}^{2}$ represents the output of the module $\mathrm{i} \in[1, \ldots, \mathrm{n}] ; \mathrm{k}_{\mathrm{i}}$ indicates the coefficient of continuation of the module $i ; h_{i}$ represents the average variation of the output values of the module $i$. The coefficient of continuation of each module exhibits the absence/presence of the individual module, regarding the requirement of the module in the exacting scenario.

For the diagrammatic reason and makes simpler computation, so that can select to minimize the structure of the MNN and use 2 modules from the model from Fig. $4 .\left(\mathrm{k}_{1}=1 ; \mathrm{k}_{2}=1 ; \mathrm{k}_{3}=1, \ldots . . \mathrm{k}_{\mathrm{n}}=0\right)$

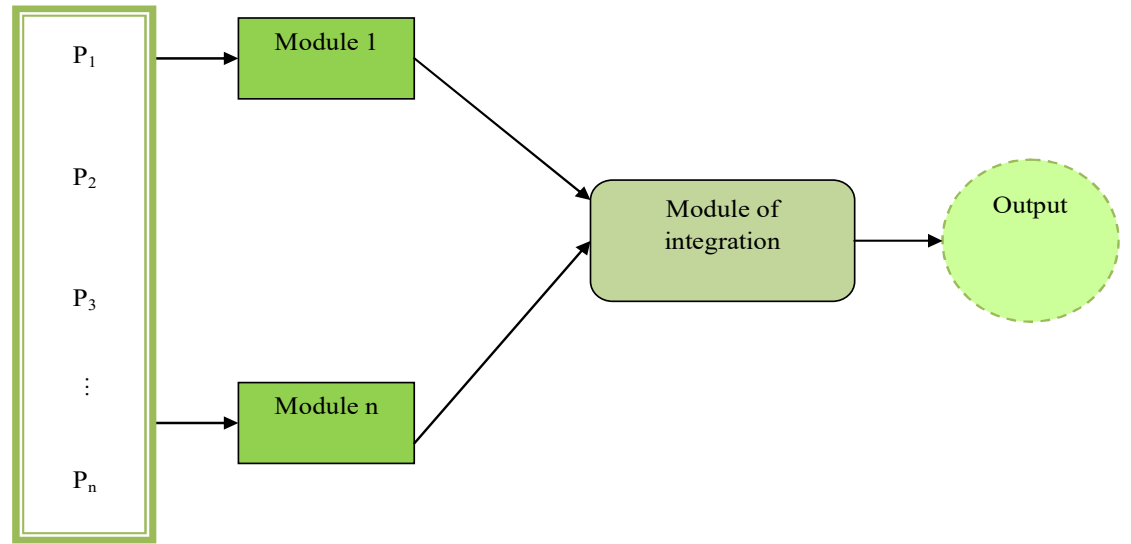

Fig. 4. Block diagram of modular neural network with 2 modules

In the first module contemplates all independent inputs (dissonance, weak dissonance, and strong dissonance). The second module observes the inputs which possess negative consonance, strong negative consonance, and weak negative consonance, positive consonance, and strong positive consonance and weak positive consonance.

In the second module, so that can minimize a few of the inputs if they have strong positive consonance. In this scenario, so that can eradicate one of the inputs. In the scenario in which they possess strong negative consonance; it refers that it has the ability in addition to eliminating a few of the inputs. Erstwhile configurations for the modular NN are potential, based on how the inputs are chosen.

\subsection{Enhanced Whale Optimization Algorithm (EWOA)}

In the conventional WOA [29], the present optimal solution is contemplated as the aim of other search experts. Then, all prey endeavors to alter their circumstances to nearby the optimal agent, and this flowchart demonstrates in Fig. 3. As the region of the optimal hunt, space is not recognized from the previous; this process of update might consequence in being trapped in neighborhood optima for the severity detection procedure.

\section{(i) Updating process of whales:}

\section{a. Encircling prey}

Humpback whale includes the prey by after that revitalizes its circumstances to the best course of action all by expanding the number of iteration from preliminary to the optimal number of iteration; it is shown in eq. (17).

$$
\mathrm{EC}=\left|\overrightarrow{\mathrm{x}} \cdot \mathrm{P}^{*}(\mathrm{t})-\mathrm{P}(\mathrm{t})\right| \text { and } \mathrm{P}(\mathrm{t}+1)=\mathrm{P}^{\text {optimal }}(\mathrm{t})-\mathrm{G} \overrightarrow{\mathrm{x}}_{1}
$$

In eq. (17), $G$ and $\vec{x}$ indicates the coefficient vectors, $t$ represents the current iteration, $P$ indicates the position on a vector of the optimal arrangement obtained in this manner, * indicates the position vector, and - indicates the element by element multiplication and mod function. The eq. (18) is used to compute the coefficient.

$$
\overline{\mathrm{x}}_{1}=2 \overline{\mathrm{x}}_{2} \cdot \overrightarrow{\mathrm{r}}-\overline{\mathrm{x}}_{2} \text { and } \mathrm{x}=2 . \mathrm{r}
$$

In eq. (18) $\mathrm{r} \rightarrow$ indicates a random vector in $(0,1)$ and $\overline{\mathrm{x}}_{2} \rightarrow$ linearly reduced from 2 to 0 during iterations.

i. Shrinking encircling strategies

This procedure is used to decrease linearly the value of $\overline{\mathrm{x}}_{2}$ from 0 to 2 ; the arbitrary value of vector $\mathrm{k}_{1}$ ranges among -1 and 1 . 
ii. Spiral updating location

A spiral circumstance is subsequently done among the circumstances of prey and whale to duplicate the helix-shaped enhancement of humpback whales as gets following.

$$
\mathrm{P}(\mathrm{t}+1)=\overrightarrow{\mathrm{G}} \cdot \mathrm{e}^{\mathrm{at}} \cdot \cos (2 \Pi \mathrm{s})+\overrightarrow{\mathrm{P}}
$$

Eq. (19) indicates the separation procedure of $i^{\text {th }}$ prey and a as the steady-state logarithmic spiral. In order to demonstrate, therefore, accept that there is a probability of picking a half among both the surrounding contracting system and the spiral model to remodel the circumstances of whales at the time of upgrade. The design gets subsequent to using eq. (20).

$$
\overrightarrow{\mathrm{P}}(\mathrm{t}+1)=\left\{\begin{array}{l}
\overrightarrow{\mathrm{P}}(\mathrm{t})-\mathrm{x}_{1} \cdot \overrightarrow{\mathrm{G}} \quad \text { if } \mathrm{i}<0.5 \\
\overrightarrow{\mathrm{G}} \cdot \mathrm{e}^{\mathrm{as}} \cdot \cos (2 \pi \mathrm{s})+\mathrm{P}(\mathrm{t}) \text { if } \mathrm{i} \geq 0.5
\end{array}\right.
$$

In eq. (20) $\mathrm{s}$ indicates an arbitrary value among -1 and $1, \mathrm{a} \rightarrow$ constant, and $\mathrm{i} \rightarrow[0,1]$. To show this synchronous behavior, expect that there is a probability of short to choose among both the enveloping spiral model and contracting system to revitalize the circumstances of whales in the middle of improvement. The theoretical model is based upon the associated as below:

\section{b) Searching for prey exploration stages: improved process}

A related method called the selection of the $\mathrm{x}_{1}$ vector can be exploited to appear for prey (examination). Definitely, humpback whales appear haphazard as shown using the location of each other.

$$
\overrightarrow{\mathrm{G}}=\left|\mathrm{x} \cdot \overrightarrow{\mathrm{P}}_{\text {rand }}-\overrightarrow{\mathrm{P}}\right| \text { and } \mathrm{P}(\mathrm{t}+1)=\frac{\mathrm{P}_{\text {rand }}-\mathrm{x}_{1} \cdot \overrightarrow{\mathrm{G}}}{\text { Correlation }}
$$

This method and $\mathrm{x}_{1}>1$ highlight exploration and permit the WOA technique to carry out a global optimum and $\mathrm{x}_{1}<1$ for updating the location of the search agents. The improvement factor creates the whales move in small steps to the prey to explore the search space economically.

c. Termination condition:

The update process of EWOA will be repeated until obtaining the best consequences. Hence, it enables any investigation operator to update its circumstances in the area of the present optimal arrangement and imitates encircling the prey all the high effectual. Fig. 5 demonstrates the flowchart of the proposed EWOA algorithm.

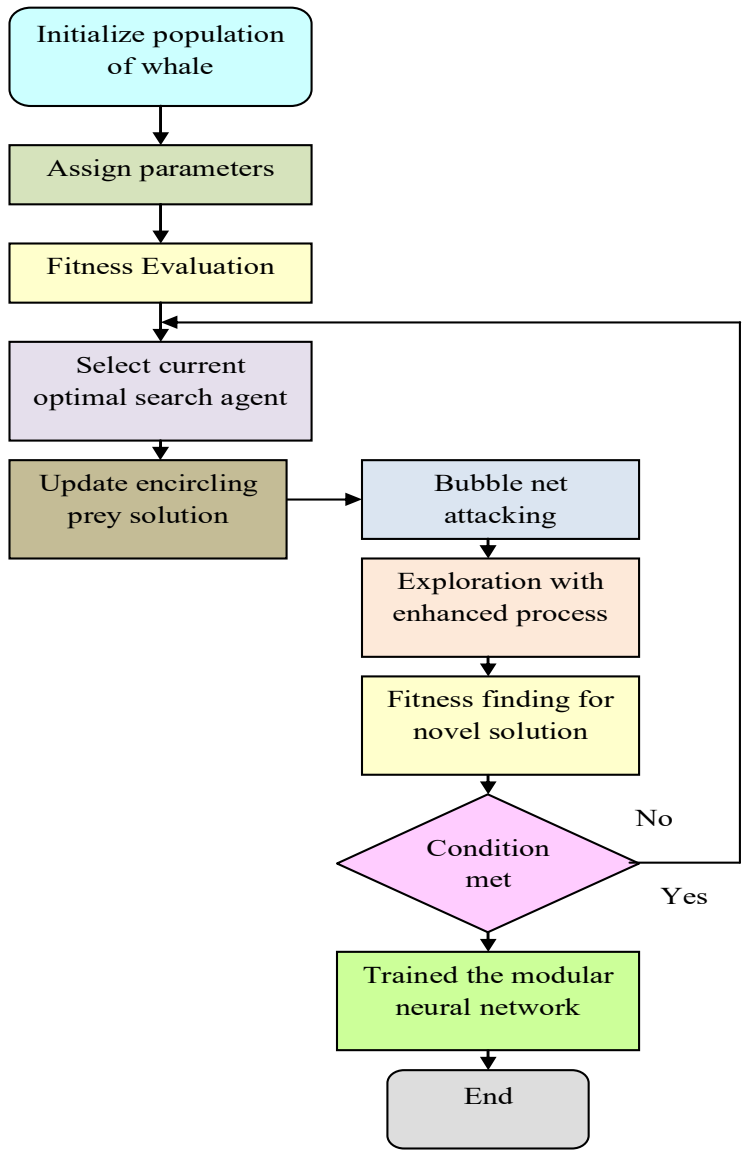

Fig. 5. Flow chart of the proposed Enhanced-WOA algorithm 


\subsection{Identification of Infection Level}

Once the proposed EWOA- MNN classifier decides the level, the subsequent step is the recognition of infection level. The infection level is decided on the basis of the output created using the proposed EWOA- MNN, entropy function and density ratio. The result produced from infection level classification is normal, level 1 or level 2. The calculation of infection level based upon the entropy function by exploiting the severity level and entropy. The entropy is explained as the conditional entropy of data series with infinite length. The entropy is exploited for measuring the uniformity of data series for predicting the new samples by empirical observations. The entropy leads to be 0 if the data sequence is periodic. Eq. (22) is used to calculate the infection level.

$$
\mathrm{IL}=\left[\frac{\mathrm{N}^{\mathrm{g}}}{\left(\mathrm{Q}_{\mathrm{c}} \times \mathrm{M}_{\mathrm{d}}\right)}+\left(-\sum_{\mathrm{g}=1}^{2} \mathrm{~L}_{\mathrm{g}} \log \mathrm{L}_{\mathrm{g}}\right)+\frac{\mathrm{S}_{\mathrm{l}}}{\mathrm{T}_{\mathrm{l}}}\right] \times \frac{1}{3}
$$

In eq. (22), $\mathrm{Q}_{\mathrm{c}} \times \mathrm{M}_{\mathrm{d}}$ signifies the density, $\mathrm{g}=1$ signifies the bacilli pixel, $\mathrm{N}^{\mathrm{g}}$ signifies the number of bacilli pixels, $\mathrm{h}=2$ signifies non-bacilli pixel, $\mathrm{S}_{1}$ signifies the severity level, $\mathrm{P}_{\mathrm{g}}$ signifies the probability of $\mathrm{g}^{\text {th }}$ pixel, and $\mathrm{T}_{1}$ signifies total levels (3).

\section{Results and Discussions}

The results and analysis of the proposed steganography method using the Haar wavelet are proposed in this section and the analysis is progressed based on the performance measures that validate the quality of the embedded image.

\subsection{Experimental Procedure}

In this section, the results achieved by the proposed algorithm were elaborated. The results were attained from Ziehl-Neelsen Sputum smear Microscopy image DataBase (ZNSM-iDB) database [31] and performance metrics, like accuracy, True Positive Rate (TPR) and False Positive Rate (FPR), were used for the analysis. Moreover, the proposed approach was compared with the conventional models such as Support Vector Machine (SVM), Neural Network (NN), Convolutional Neural Network (CNN), Random Forest (RF), and Support Vector Neural Network (SVNN).

\subsection{Statistical Analysis}

In Fig. 6, the analysis of the proposed algorithm for TB classification by exploiting the Training percentage is demonstrated. From the Fig 6, the performance of the proposed model is $22 \%$ better than the SVM, 19\% better than NN, 17\% better than CNN, 13\% better than SVNN algorithm. Here, the FPR value is minimum, which is exhibited by the proposed model as compared to conventional models.

The performance analysis of the proposed algorithm for TB classification by exploiting the cross-fold percentage is shown in Fig 7. Here, the TPR value attained is high as compared with the conventional algorithms. Fig. 8 shows the performance analysis of the proposed algorithm for severity analysis by exploiting the Training percentage. In Fig. 9, the performance analysis of the proposed algorithm for severity analysis by exploiting the Training percentage is exhibited. Here, the performance of the proposed model is 33\% better than the SVM, 29\% better than NN, 25\% better than CNN, $21 \%$ better than SVNN algorithm.

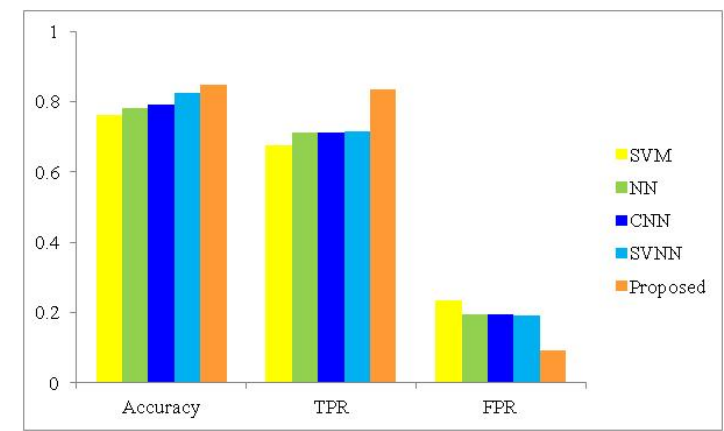

Fig. 6. Analysis of the proposed algorithm for TB classification by exploiting the Training percentage 


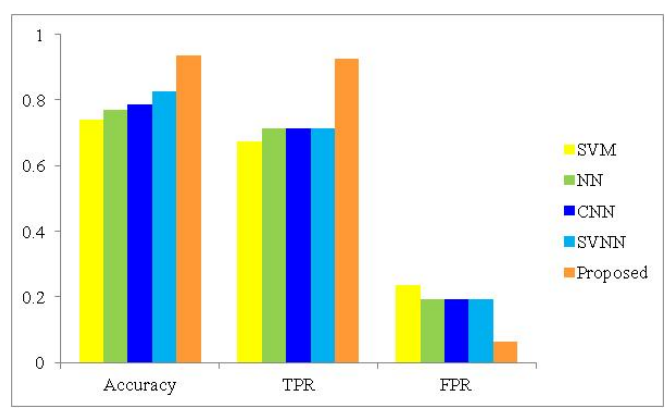

Fig. 7. Analysis of the proposed algorithm for TB classification by exploiting the cross-fold validation

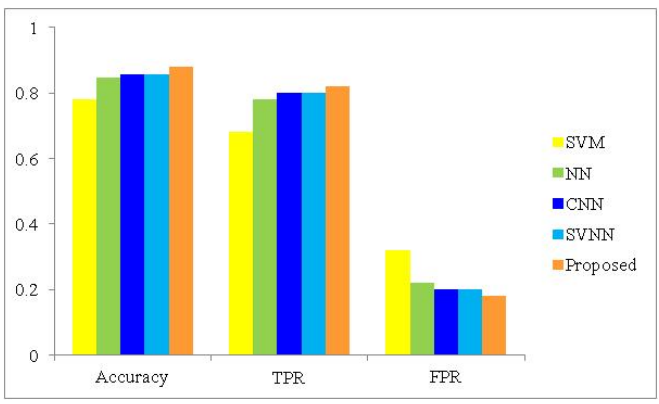

Fig. 8. Analysis of the proposed algorithm for severity analysis by exploiting the Training percentage

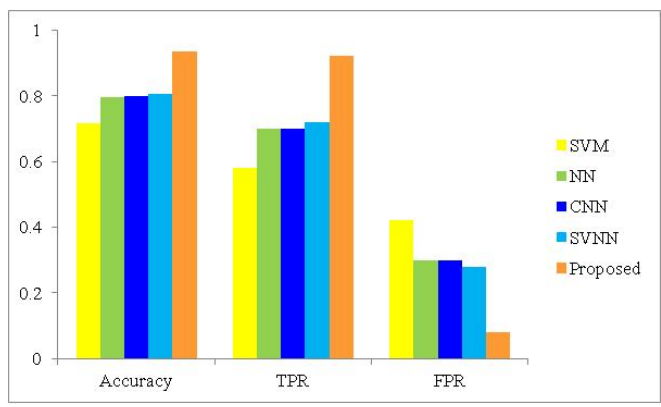

Fig. 9. Analysis of the proposed algorithm for severity analysis by exploiting the cross-fold validation

\section{Conclusion}

In this paper, an approach for severity infection and detection level recognition exploiting the modular neural network classifier. At first, the TB patients are recognized using WOA-CNN that use WOA technique for training the CNN. Subsequently, for severity analysis, the feature extraction procedure was performed on the basis of the features, such as a number of bacilli, area of bacilli, overlapping bacilli, skeleton features, and scattering coefficients. The extracted features were utilized by the proposed EWOA-MNN for the severity evaluation. The proposed algorithm was modeled by exploiting the proposed EWOA approach that is the novel method by enhancing the WOA approach. Hence, the proposed EWOA-MNN does the severity detection for evaluating the TB and on the basis of the severity evaluation, the infection level was determined. The testing was performed by exploiting the ZNSM-iDB database and the performance of the conventional and proposed algorithm was analyzed by exploiting accuracy rate, FPR, and TPR values. Finally, the performance of the proposed algorithm exhibits better performance for TPR, accuracy, and FPR for the first level and second level classification.

\section{Compliance with Ethical Standards}

Conflicts of interest: Authors declared that they have no conflict of interest.

Human participants: The conducted research follows the ethical standards and the authors ensured that they have not conducted any studies with human participants or animals. 


\section{References}

[1] Jie Wu, Chongguang Yang, Liping Lu, Wanqin Dai,"Detection of tuberculosis laboratory cross-contamination using whole-genome sequencing",Tuberculosis, Volume 115, March 2019, Pages 121-125.

[2] Ellen Jo Baron, Fred C. Tenover, Devasena Gnanashanmugam,"Direct Detection of Mycobacterium tuberculosis in Clinical Specimens Using Nucleic Acid Amplification Tests", Clinical Microbiology Newsletter, Volume 40, Issue 13, 1 July 2018, Pages 107-112.

[3] Jeewan Thapa, Bhagwan Maharjan, Meena Malla, Yukari Fukushima, Yasuhiko Suzuki,"Direct detection of Mycobacterium tuberculosis in clinical samples by a dry methyl green loop-mediated isothermal amplification (LAMP) method",Tuberculosis, Volume 117, July 2019, Pages 1-6.

[4] Karoline Wagner, Benjamin Preiswerk, Peter M. Keller, Bettina Schulthess,"Defining threshold values in microscopic examination and qPCR for optimal performance of line probe assays for resistance detection in Mycobacterium tuberculosis complex", Diagnostic Microbiology and Infectious Disease, Volume 95, Issue 2, October 2019, Pages 159-161.

[5] Reza Kamali Kakhki, Alireza Neshani, Mojtaba Sankian, Kiarash Ghazvini, Mahsa Sayadi,"The short-chain dehydrogenases/reductases (SDR) gene: A new specific target for rapid detection of Mycobacterium tuberculosis complex by modified comparative genomic analysis",Infection, Genetics and Evolution, Volume 70, June 2019, Pages 158-164.

[6] Park JE, Huh HJ, Koh WJ, Song DJ, Ki CS, Lee NY. Performance evaluation of the Cobas TaqMan MTB assay on respiratory specimens according to clinical application. Int J Infect Dis 2017;64:42-6.

[7] Chen JH, She KK, Kwong TC, Wong OY, Siu GK, Leung CC, et al. Performance of the new automated Abbott RealTime MTB assay for rapid detection of Mycobacterium tuberculosis complex in respiratory specimens. Eur J Clin Microbiol Infect Dis 2015;34:1827-32.

[8] Hofmann-Thiel S, Molodtsov N, Antonenka U, Hoffmann H. Evaluation of the Abbott RealTime MTB and RealTime MTB INH/RIF assays for direct detection of Mycobacterium tuberculosis complex and resistance markers in respiratory and extrapulmonary specimens. J Clin Microbiol 2016;54:3022-7.

[9] Nathavitharana RR, Cudahy PG, Schumacher SG, Steingart KR, Pai M, Denkinger CM. Accuracy of line probe assays for the diagnosis of pulmonary and multidrug-resistant tuberculosis: a systematic review and metaanalysis. Eur Respir J 2017;49:1601075.

[10] Huyen MN, Tiemersma EW, Lan NT, Cobelens FG, Dung NH, Sy DN, et al. Validation of the GenoType MTBDRplus assay for diagnosis of multidrug resistant tuberculosis in South Vietnam. BMC Infect Dis 2010;10:149.

[11] Gu Y, Wang G, Dong W, Li Y, Ma Y, Shang Y, et al. Xpert MTB/ RIF and GenoType MTBDRplus assays for the rapid diagnosis of bone and joint tuberculosis. Int J Infect Dis 2015;36:27-30.

[12] Obasanya J, Lawson L, Edwards T, Olanrewaju O, Madukaji L, Dacombe R, et al. FluoroType MTB system for the detection of pulmonary tuberculosis. ERJ Open Res 2017;3:00113-2016.

[13] Hofmann-Thiel S, Hoffmann H. Evaluation of Fluorotype MTB for detection of Mycobacterium tuberculosis complex DNA in clinical specimens from a low-incidence country. BMC Infect Dis 2014;14:59.

[14] Nikam C, Jagannath M, Narayanan MM, Ramanabhiraman V, Kazi M, Shetty A, et al. Rapid diagnosis of Mycobacterium tuberculosis with Truenat MTB: a near-care approach. PLoS One 2013;8:e51121.

[15] WHO. The use of loop-mediated isothermal amplification (TBLAMP) for the diagnosis of pulmonary tuberculosis: policy guidance. World Health Organization; 2016.

[16] Luo RF, Scahill MD, Banaei N. Comparison of single-copy and multicopy real-time PCR targets for detection of Mycobacterium tuberculosis in paraffin-embedded tissue. J Clin Microbiol 2010;48:2569-70.

[17] Schewe C, Goldmann T, Grosser M, Zink A, Schluns K, Pahl S, et al. Inter-laboratory validation of PCR-based detection of Mycobacterium tuberculosis in formalin-fixed, paraffin-embedded tissues. Virchows Arch 2005;447:573-85.

[18] Lin SY, Rodwell TC, Victor TC, Rider EC, Pham L, Catanzaro A, et al. Pyrosequencing for rapid detection of extensively drug-resistant Mycobacterium tuberculosis in clinical isolates and clinical specimens. J Clin Microbiol 2014;52:475-82.

[19] Derek Bradley and Gerhard Roth, "Adaptive Thresholding using the Integral Image", vol.12, no.2, pp.13-21, Jan 2011.

[20] Anitha Mary M. O. Chacko and P. M. Dhanya, " A Comparative Study of Different Feature Extraction Techniques for Offline Malayalam Character Recognition", Computational Intelligence in Data Mining, vol.2, pp.9-18, 2014.

[21] SzabolcsSergyan, " Color histogram features based image classification in content-based image retrieval systems", In Proceedings of the 6th International Symposium on Applied Machine Intelligence and Informatics, pp.221 - 224, 2008.

[22] Jabid, T., Kabir, M.H. and Chae, O., "Gender classification using local directional pattern (LDP)", 20th International Conference on Pattern Recognition (ICPR) IEEE, pp. 2162-2165, August 2010.

[23] Bongjin Jun; Inho Choi; Daijin Kim, "Local Transform Features and Hybridization for Accurate Face and Human Detection", IEEE Transactions on Pattern Analysis and Machine Intelligence, vol.35, no.6, pp.1423 1436, 2013.

[24] Deegalla, S. and Bostrom, H., "Reducing high-dimensional data by principal component analysis vs. random projection for nearest neighbor classification," In proceedings of International Conference on Machine Learning and Applications, pp. 245-250, December 2006. 
[25] Yang, C., Tiebe, O., Shirahama, K. and Grzegorzek, M., "Object matching with hierarchical skeletons," Pattern Recognition, vol.55, pp.183-197, 2016.

[26] Pandey, P., Singh, R. and Vatsa, M., "Face recognition using scattering wavelet under Illicit Drug Abuse variations," In ICB, pp. 1-6, June 2016.

[27] P. Melin, Modular Neural Networks and Type-2 Fuzzy Systems for Pattern Recognition, vol. 389, Springer, Berlin, Heidelberg, 2012.

[28] M. Hagan, H. Demuth, and M. Beale, Neural Network Design,PWS Publishing, Boston, MA, 1996.

[29] Mirjalili S, Lewis A. The whale optimization algorithm. Adv Eng Softw. 2016;95:5167.

[30] Qian S, Liu H, Liu C, Wu S, Wong HS (2018) Adaptive activation functions in convolutional neural networks. Neurocomputing, 272:204-212. ISSN 0925-2312.

[31] RS, C., "Fractional Crow search-based Support Vector Neural Network for patient classification and severity analysis of Tuberculosis," IET Image Processing, 2018.

[32] J.S. Anita and J.S. Abinaya,"Impact of Supervised Classifier on Speech Emotion Recognition", Multimedia Research, Volume 2, Issue 1, January 2019. 